\title{
RIPARTIRE DAL LAVORO PUBBLICO \\ INTRODUZIONE
}

di Alberto Mattei e Vladimiro Soli

La scelta di dedicare ai cambiamenti del mondo della Pubblica Amministrazione la parte monografica del primo numero 2016 di Economia e società regionale può apparire, per molte ragioni, scontata. In realtà, il dibattito sul ruolo del pubblico nel nostro Paese non offre sovente l'opportunità di sviluppare una riflessione di ampio respiro. Il tema costituisce un elemento ricorrente di divisione, anche analitica, con contrapposizioni ideologiche e difese di interessi di parte che fanno prevalere le polemiche di schieramento piuttosto che le soluzioni. L'urgenza di tali questioni tende tuttavia a riproporsi periodicamente, resa più acuta dal maturare di spinte che mettono in tensione il funzionamento corrente delle strutture di servizio pubblico. La ritrovata centralità del problema Pubblica Amministrazione ha di questi tempi il pregio di richiedere strategie di risposta non autoreferenziali, che sappiano guardare ai bisogni della società come leva d'innesto di qualsiasi processo di riforma. La crisi è oggi tanto acuta da poter divenire una grande opportunità per l'avvio di un rinnovamento complessivo della Pubblica Amministrazione che consenta di costruire un nuovo incontro tra necessità concrete dei cittadini e servizi pubblici.

Lo stato di necessità in cui versa la Pubblica Amministrazione ha trovato una risposta del Governo Renzi nel tentativo di promuovere un processo di modernizzazione che, a suo dire, dovrebbe mettere il Paese al passo con le trasformazioni generali. Nel merito dell'assortita serie di provvedimenti fin qui assunti, molti interventi risultano più comprensibili per le motivazioni, dichiarate o sottese, che per la efficacia e prevedibilità delle possibili risultanze. Allo stato, si può notare che si è in presenza (dell'ennesimo) intervento legislativo, peraltro condotto con un'assertività che rischia di indurre qualche sottovalutazione dei problemi, anche per la scarsa disponibilità a riflettere sui limiti delle azioni riformatrici 
precedenti. Con una disinvoltura forse eccessiva, ci si è avviati sulla strada di una presunta sufficienza performativa degli strumenti legislativi, fidando su una serie di misure considerate così efficaci da incidere in modo penetrante sui mali antichi della Pubblica Amministrazione. Nonostante l'uso di tambureggianti strategie mediatiche, rimane qualche dubbio che un'amministrazione con tare antiche e recenti possa essere rimessa in moto affidandosi a sbrigative ricette aziendalistiche: meno costi, meno tempi, semplificazione. Resta l'impressione di un Governo che non sembra essere scalfito da dubbi sul fatto di essere sulla strada giusta, così come esso non fa mistero dell'intenzione di spazzare via le forme di resistenza, fannulloni, assenteisti, dirigenti imbelli (sindacati?), né di rinnovare la propria convinzione di riuscire ad avviare mutamenti forti a costi irrisori (vedi la voce: contratti).

La sensazione complessiva suscitata dalle dichiarazioni governative sui primi abbozzi del disegno riorganizzativo, quindi, è di un'apparente incomprensione dei nodi che frenano l'innovazione amministrativa. Molti provvedimenti sembrano slegati tra di loro e, se anche possono essere adeguati in casi specifici, faticano a esprimere un disegno riformatore coerente. È perfino superfluo dire che non giovano le ripetute manifestazioni di determinismo pseudo-autoritario, utili più a soddisfare le attese di un'opinione pubblica esasperata, che a dare sistemazione adeguata ai problemi (es. il decreto sui cosiddetti "furbetti del cartellino"). Infine, occorre riconoscere che non si mostra di tenere in gran conto le difficoltà dell'azione, soprattutto il mondo del lavoro pubblico non viene considerato un possibile alleato in questa operazione di rinnovamento, e alla mancanza del suo consenso e approvazione, si contrappone la ricerca di sostegno da parte di altri referenti.

Se questi motivi giustificano la preoccupazione del sindacato per come si affronta il tema del lavoro pubblico, oggi non è più possibile fermarsi a una pur necessaria visione critica delle impostazioni governative. Lo stato della Pubblica Amministrazione richiede al sindacato, anche per i segni della sua difficoltà nella rappresentanza settoriale, una riflessione meno scontata e prevedibile. Più che per altri settori produttivi, l'iniziativa sindacale su questo terreno deve saper proporre una strategia di intervento ambiziosa, affrontando le sfide poste dall'innovazione dei servizi e dalla necessità di promuovere una rinnovata politica di qualità delle prestazioni pubbliche.

Questa premessa sarebbe già in grado di giustificare l'avvio di una riflessione radicale sul ruolo dell'intervento pubblico ma, nel frattempo, è venuta maturando una questione di portata ancora maggiore. Se il tema del mutamento della Pubblica Amministrazione è di grande attualità, è 
bene rilevare che si tratta di un'attualità di nuovo tipo, che non è possibile sciogliere in modo rituale. Appare urgente, piuttosto, partire dalla constatazione che in questi anni si sono venute accumulando una serie di condizioni che, a prescindere dagli obiettivi e dalle finalità del Governo, spingono per una riorganizzazione complessiva del sistema, in termini di regole, forme e pratiche di lavoro. Non va poi sottovalutato, che la nozione di lavoro pubblico è strettamente intrecciata con questioni di natura istituzionale (welfare e diritti democratici); ciò sollecita il sindacato a considerare il problema del lavoro pubblico come questione che ha a che fare con la costituzione materiale del Paese e non può essere confinata in ambiti di natura settoriale.

A fronte di un orizzonte così impegnativo, l'azione di Governo non sembra essere guidata da adeguato rigore. I segnali raccolti in questi mesi evidenziano che sul terreno dell'analisi giuridica si è di fronte a una produzione normativa che presenta aspetti non del tutto indirizzabili a una visione unitaria del lavoro pubblico e a una precisa visione di insieme, se non per grandi linee di indirizzo volte alla semplificazione, alla trasparenza e alla digitalizzazione. Lo stesso si può dire per i primi decreti attuativi di gennaio 2016, nei confronti dei quali vi è chi ha ravvisato, nel complesso, correttivi di rotta non sempre coerenti, più che una vera e propria riforma (Olivieri, 2016).

Va da sé che in una prospettiva d'insieme, il processo di riforme attuate in materia, partito all'inizio degli anni Novanta, è stato caratterizzato da stratificazioni, elementi di alluvionalità e «continue discontinuità ordinamentali, a loro volta espressione di paradigmi interpretativi e dunque di modelli cognitivi non sempre facilmente integrabili»(Viscomi, 2015: 195). Peraltro, che la Riforma Madia possa risultare più efficace della contrattualizzazione o privatizzazione delle riforme degli anni novanta, o della rilegificazione della Riforma Brunetta, è ancora troppo presto per dirlo. Se il filo conduttore della riforma targata Renzi, quindi, è quello di accelerare il passaggio verso una modernizzazione dell'amministrazione dello Stato, si deve dire che alcune premesse appaiono problematiche. Una per tutte: il fatto che in carenza di un ruolo da riconoscere ai lavoratori pubblici, come ricorda Lorenzo Zoppoli nel suo contributo (in questo numero alle pp. 12-36), si finisce per concepire il lavoro più come impedimento che come risorsa. Ne è emblema, in questo senso, all'interno di uno dei decreti di gennaio 2016, sia la modifica alla norma del Testo Unico del pubblico impiego sul licenziamento disciplinare volta ad allargare il perimetro della "falsa attestazione della presenza in servizio" con un'accelerazione della procedura di licenziamento (cd. "furbetti del cartellino"); sia l'assenza, al momento, di qualsivoglia 
decreto volto ad intervenire su quello che la delega prevede in materia di "riordino" - termine che ricorre ossessivamente più volte nella delega della disciplina del lavoro alle dipendenze della Pubblica Amministrazione come la «previsione nelle procedure concorsuali pubbliche di meccanismi di valutazione finalizzati a valorizzare l'esperienza professionale acquisita da coloro che hanno avuto rapporti di lavoro flessibile con le amministrazioni pubbliche [...]» (art. 17, co. 1 lett. a) 1. 124/15), o anche la «previsione di prove concorsuali che privilegino l'accertamento della capacità dei candidati di utilizzare e applicare a problemi specifici e casi concreti nozioni teoriche, con possibilità di svolgere unitariamente la valutazione dei titoli e le prove concorsuali relative a diversi concorsi» (art. 17, co. 1 lett. b) 1. 124/15).

Si tratta di profili certamente delicati, e da dosare con cura e attenzione, ma il messaggio mediatico, almeno per il momento, è chiaro: prima si punisce in un'ottica repressiva chi sbaglia, poi si ragiona e si interviene con una normativa di sistema su come rendere migliore, nella qualità, la macchina amministrativa, dal punto di vista del personale di chi vi lavora già con rapporti flessibili o di chi vi vuole accedere.

Ancora più rilevante sul versante giuridico, risulta l'assenza di ogni indirizzo politico sul ruolo da attribuire alla contrattazione all'interno di questo processo riformatore. Paradossalmente, la delega si limita a un paio di richiami sulla contrattazione integrativa e sul ruolo di supporto dell'Aran, proprio nel momento in cui vengono ridotti a quattro i comparti del pubblico impiego e nonostante la pronuncia della Corte costituzionale sulla illegittimità costituzionale del regime di sospensione della contrattazione collettiva

Qualora si concordi sulla legittimità di queste preoccupazioni, appaiono evidenti le ragioni che ci hanno spinto a dedicare il tema monografico di Esr ai problemi del lavoro nella PA. La portata di questa sfida attesta in modo evidente che il tema impone di rifuggire da visioni un po' pigre, limitandosi a immaginare una qualche manutenzione dell'esistente. Si è quindi cercato di tematizzare questioni che sono destinate a operare nel profondo della Pubblica Amministrazione, guardando ai segni di una trasformazione sintomatica delle tensioni che pervadono l'intera dimensione del lavoro pubblico. Pur non volendo caricare i contributi della rivista di compiti analitici troppo ambiziosi, è parso necessario fare uno sforzo per inoltrarsi lungo nuove piste di ricerca, valutando tutti quei segnali che ci mettono in guardia sulla necessità di cogliere al meglio cambiamenti e discontinuità. Se di alcune dinamiche in corso il disegno ispiratore appare immediatamente percepibile, in altri casi si tratta ancora di osservare dei processi che sollecitano a distinguere tra ciò che è in via di 
affermazione e ciò che è destinato a rimanere inerte. In ogni caso, districando la matassa nei suoi fili principali, appare evidente che dietro l'agitarsi di molti interventi continua a operare l'obiettivo di modificare il ruolo tradizionalmente riconosciuto all'azione pubblica. La logica con cui si punta a modernizzare il funzionamento della Pubblica Amministrazione sembra ricalcare tendenze praticate altrove con esiti problematici, soprattutto in termini di rispetto delle attese dei cittadini e di partecipazione democratica. Il disegno di riorganizzazione dei compiti dell'intervento pubblico ha molti aspetti mostrandosi principalmente volto a favorire indirizzi che ridimensionano il ruolo del servizio pubblico e rischiano di perpetuarne il declino.

\section{I contributi di questo numero}

La proposta di temi del numero mette al centro della riflessione sindacale alcuni passaggi che ci appaiono improcrastinabili. Essi consentono di delineare un quadro interpretativo in grado di fissare le questioni dirimenti, quelle che devono stare al centro della discussione e dell'iniziativa sindacale. Non va sottaciuto che la costruzione di un numero monografico deve fare i conti anche con delle limitazioni nell'arco dei temi affrontati. Quella più importante ha riguardato l'impossibilità di contare su un'analisi della contrattazione integrativa di settore, laddove l'assenza di esperienze adeguatamente monitorate rende difficile una valutazione delle prospettive. Da oltre un lustro le scelte dei diversi governi hanno posto un limite invalicabile alla possibilità di svolgere una coerente contrattazione di secondo livello, privando il sindacato, ma anche le controparti amministrative, di un piano di confronto che si sarebbe rivelato di particolare utilità nell'attuare scelte di politica organizzativa qualificate e efficienti.

Un altro limite della nostra riflessione è relativo al mancato approfondimento della dimensione economica dei servizi pubblici: sia nel senso del contributo dei servizi alla vita economica del paese, sia nel considerare i vincoli all'azione, anche riformatrice, imposti dalla restrizione delle risorse pubbliche. È evidente che la tenuta dei sistemi di servizi pubblici, - welfare e non solo - deve fare i conti con vincoli economici sempre più stringenti. Ciò ha dato luogo a una ricca letteratura sulle soluzioni che sono chiamate a ottimizzare l'incrocio tra risorse pubbliche e sviluppo delle funzioni di servizio. Il tema è obiettivamente molto controverso, a dispetto di considerazioni generali che puntano sbrigativamente a prevedere solo la possibilità di ridurre le spese su questo terreno. Infine, non è stato possibile affrontare un altro tema che appare di grande attualità: il 
rapporto tra le esperienze, sempre più diffuse, di welfare privato e la tenuta delle forme di welfare pubblico. Oggigiorno sono numerosi gli esempi di interventi volti a contenere l'effetto conflittuale della contrattazione di posto di lavoro puntando su soluzioni di welfare privato. $\grave{E}$ abbastanza evidente che queste scelte, pur gradite ai lavoratori, ma per necessità più che per adesione, hanno il potenziale effetto di interagire con gli spazi riservati al welfare pubblico e universalistico, con implicazioni di portata ancora non ben chiara a molti.

Al netto di questi limiti, la trattazione del numero monografico segue il seguente ordine.

Il contributo di Lorenzo Zoppoli, Università Federico II di Napoli, descrive le linee di fondo dell'ennesimo progetto di riforma governativo, soffermandosi sia sulle materie, inerenti il lavoro pubblico, toccate dai primi decreti approvati a gennaio 2016 (licenziamento disciplinare e dirigenza sanitaria); sia sulle norme che riguardano il lavoro pubblico presenti nella legge delega di agosto 2015 (dirigenza pubblica con rischi sia di "iper-regolazione legislativa" e "cripto-politicizzazione"; il riordino della normativa sul lavoro pubblico in un testo unico, non privo di aspetti critici; non ultimo il ruolo non definito della contrattazione da "inaspettata" a "ostacolata"). Nel complesso, vengono messe in rilievo luci e ombre del processo riformatore, pur comunque nell'impossibilità, segnalata dall'autore, di esprimere una valutazione conclusiva e approfondita su un processo attuativo ancora in progress.

L'intervento di Gagliardo Deidda, Università di Ferrara, si misura su un tema che rappresenta un fattore di rilevante criticità per le strategie di innovazione della Pubblica Amministrazione, quello della valutazione e misurazione delle prestazioni. Il tema viene affrontato a partire da una riflessione sulle non positive esperienze maturate lungo gli anni, segnalando come l'inadeguata strutturazione di questi processi sia sovente da considerare tra le cause di fallimento dei processi legislativi. Qualsiasi ambizioso progetto di rinnovamento della Pubblica Amministrazione, infatti, ha l'onere di creare le condizioni per la realizzazione di un efficace processo di misurazione e valutazione delle prestazioni.

Il contributo che contiene un'intervista di Vladimiro Soli a Ota De Leonardis, Università di Milano Bicocca, apre a una riflessione di più lungo orizzonte sui cambiamenti che investono la condizione lavorativa all'interno del settore pubblico. La conversazione si preoccupa di segnalare come stiano mutando anche i riferimenti generali su cosa deve essere effettivamente considerato lavoro pubblico, lungo processi di trasformazione che ne modificano la stessa configurazione settoriale. Si tratta di un approfondimento che, affrontando il tema nella sua dimensione di tipo 
più sociologico (come si sta in un'organizzazione pubblica in cambiamento), sposta l'attenzione sulle trasformazioni strutturali che hanno investito il lavoro pubblico in un complicato percorso di svuotamento e delegittimazione, all'origine di tanta parte dei problemi di efficienza e qualità dei servizi.

A questi tre contributi provenienti dall'ambito universitario, seguono quelli dal mondo sindacale. L'intervento di Michele Gentile, del Dipartimento Settori Pubblici della Cgil, offre l'occasione per soffermarsi sul campo ristretto entro cui si muove il sindacato, schiacciato tra politiche di riforma sbrigative e semplificatrici, e difficoltà e disillusioni dei lavoratori. La sfida è di ritrovare nelle risorse del sindacato, confronto con i lavoratori e strumenti di partecipazione attiva, gli elementi che possono permettere di divenire interlocutori obbligati di ogni processo di cambiamento.

Sempre in ottica sindacale, il Segretario Generale della FP Cgil Veneto Daniele Giordano affronta alcuni dei temi che interrogano direttamente le politiche di una struttura regionale di categoria. Per rilevanza e complessità il comparto sanitario appare un campo emblematico della crescente difficoltà del pubblico a soddisfare le esigenze dei cittadini. La possibilità di confrontarsi con il soggetto direttamente responsabile del servizio, la Regione, obbliga/consente al sindacato di porsi come interlocutore attivo delle strutture di governo del sistema, impegnandosi nell'attivazione di una strategia che sappia collegare la tutela e la valorizzazione del lavoro con una certezza di qualità del servizio per tutti $\mathrm{i}$ cittadini.

Infine, Rosa Pavanelli, Segretaria Generale dell'Internazionale dei Servizi Pubblici (ISP/PSI), si interroga sul futuro della rappresentanza e della partecipazione dei lavoratori europei in ambito pubblico.

\section{Riferimenti bibliografici}

Viscomi A. (2015). Lavoro pubblico: cose fatte, disfatte e da fare. Il Lavoro nelle Pubbliche Amministrazioni, 2: 195-215.

Olivieri L. (2016). La raffica di decreti che corregge ma non riforma la PA, Lavoce.info, 22 gennaio $<$ www.lavoce.info $>$. 